\title{
Behavioral responses to combinations of timed light, food availability, and ultradian rhythms in the common vole (Microtus arvalis)
}

\author{
Daan R. van der Veen ${ }^{1,2}$, Dirk-Jan Saaltink ${ }^{1,3}$, and Menno P. Gerkema ${ }^{1}$ \\ ${ }^{1}$ University of Groningen, Department of Chronobiology \\ Nijenborgh 7, PO Box 11103, NI 9747 AG Groningen, The Netherlands \\ ${ }^{2}$ University of Notre Dame, Department of Biological Sciences \\ Galvin Life Science Center, Notre Dame, IN 46556, USA \\ ${ }^{3}$ Present address: University Leiden, Medical Pharmacology \\ LACDR, Einsteinweg 55, 2300 RA Leiden, The Netherlands
}

\section{Corresponding author:}

Daan van der Veen

University of Notre Dame

Biological Sciences

242 Galvin Life Sciences

Notre Dame, IN 46556-5645 USA

Email: vanderveen@nd.edu

Phone: +1 574 631-1869

Abbreviations:

CG: Centre of Gravity, FA: food Availability, LD: Light-Dark, LP: Light Pulse, SCN: Suprachiasmatic Nuclei, T: Period of external Zeitgeber cycle; LEO: Light Entrainable Oscillator, FEO: Food Entrainable Oscillator 


\section{ABSTRACT}

Light is the main entraining signal of the central circadian clock, which drives circadian organization of activity. When food is made available during only certain parts of the day, it can entrain the clock in the liver without changing the phase of the central circadian clock. While a hallmark of food entrainment is a behavioral anticipation of food availability, the extent of behavioral alterations in response to food availability has not been fully characterized. We have investigated interactions between light and temporal food availability in the timing of activity in the common vole. Temporally restricted food availability enhanced, or attenuated, re-entrainment to a phase advance in light entrainment when it was shifted together with the light or remained at the same time of day, respectively. When light entrained behavior was challenged with temporal food availability cycles with a different period, two distinct activity components were observed. More so, our data indicate that in the presence of cycles of different period length of food and light, an activity component emerged that appeared to be driven by a free-running (light-entrainable) clock. Because we have previously shown that in the common vole altering activity through running-wheel availability can alter the effectiveness of food availability to entrain the clock in the liver, we included running-wheel availability as a parameter that alters the circadian/ultradian balance in activity. In our protocols, running-wheel availability enhanced the entraining potential of both light and food availability in a differential way. The data presented here show that in the vole activity is a complex of individually driven components and that this activity is, itself, an important modulator of the effectiveness of entraining signals such as light and food. (Author correspondence: vanderveen@nd.edu)

Keywords: Entrainment, Food restriction, Light, Zeitgeber, Running wheel, Ultradian 


\section{INTRODUCTION}

Overt circadian rhythmicity in behavior has been shown to be driven by the clock in the suprachiasmatic nuclei ( $\mathrm{SCN}$ ) of the hypothalamus. After lesioning of the $\mathrm{SCN}$, circadian rhythmicity of behavior and physiology disappears (Moore \& Eichler, 1972; Stephan \& Zucker, 1972), but it can be restored by transplantation of (fetal) SCN tissue (Ralph et al., 1990). The major Zeitgeber for the SCN is light, and the SCN is regarded as being part of the Light Entrainable Oscillator (LEO). Animals can also entrain their behavior to cycles in food availability (Castillo et al., 2004; Mistlberger, 1993; Stephan, 1986). Stephan (1979) showed that this also holds true for SCN-lesioned rats, and since then food availability has gained interest as a Zeitgeber for a self-sustained oscillator system outside the SCN.

Temporal food availability has thus been shown to be an alternative Zeitgeber. Krieger et al. (1977) reported circadian rhythms in plasma corticosteroid levels and body temperature in rats that were entrained by a regime of $2 \mathrm{~h}$ of food/day. Timing of feeding (itself normally in phase with other behaviors that are under the control of the SCN) synchronizes the liver's circadian clock, without altering phase of the LEO in the SCN (Damiola et al., 2000; Stokkan et al., 2001). Typically, when an animal is subjected to feeding schedules, a rise in locomotor activity is seen prior to the restoration of the food availability, and this food anticipatory activity is one of the main arguments for an oscillator underling food entrainment (the Food Entrainable Oscillator [FEO], for review see Mistlberger, 2009; Stephan, 2002).

We have previously shown that in the common vole (Microtus arvalis), the molecular clock in the liver does not exhibit circadian oscillations of clock genes under ad libitum feeding conditions, but that these can be induced by restricting food availability to a certain part of the day and/or providing a running wheel (Van der Veen et al., 2006). The vole is an ultradian forager, feeding every 2-3 h (Daan \& Slopsema, 1978; Gerkema \& van der Leest, 1991), but by making a running wheel available to these voles, a circadian activity component is emphasized (Gerkema et al., 1990; Van der 
Veen et al., 2006). Using hypothalamic lesions, it has been show that it is not the SCN, but the retrochiasmatic area of the hypothalamus which is needed for the generation of these ultradian behavioral rhythms (Gerkema et al., 1990). The rather weak circadian system in voles, even resulting in a loss of circadian rhythmicity in constant dim light, has been shown to be accompanied by low amplitudes of transcriptional activity of clock-related genes of the SCN (Van der Veen et al., 2008). The attenuating effect of an ultradian behavioral rhythm on the food-entrainable clock in the liver, which occurred without a running wheel and under ad libitum feeding conditions, raises questions about the nature of the interaction of activity and food and light entrainment in the vole.

Effects of activity on timing of circadian activity have been shown under different conditions. Rodents have been entrained to daily activity bouts elicited by cage cleaning (Mrosovsky, 1988) or forced running (Marchant \& Mistlberger, 1996; Mistlberger, 1991) as well as voluntary running (Edgar \& Dement, 1991) in a wheel. In hamsters, re-entrainment to a phase advance in light is sped up by inducing running-wheel activity prior to phase advancing the LD cycle (Mrosovsky \& Salmon, 1987), and such timing of activity can also change phase of entrainment (Mistlberger \& Holmes, 2000). In hamsters, the ability to be active is essential for behavioral phase shifting in response to a dark pulse (Reebs et al., 1989). These reports indicate that activity can modulate photic entrainment of behavior.

Using the vole to study the three-way interaction of light, food, and activity in driving of activity can give us new insights, because the vole switches between ultradian and circadian activity patterns, and this activity, itself, has been shown to affect food as an entraining signal for the liver clock. We used a single light pulse to entrain voles, minimizing negative masking of activity (Aschoff, 1960; Mrosovsky, 1999), and we challenged the light entrainment both by food restriction and changes in the ultradian/circadian behavioral patterns through running-wheel availability. 


\section{METHODS}

\section{Animals}

Common voles (Microtus arvalis) were bred in-house in a lab-based colony which was founded by individuals trapped in the Netherlands $\left(53^{\circ} 20^{\prime} \mathrm{N}: 6^{\circ} 16^{\prime} \mathrm{E}\right)$ and subsequently bred in-house for multiple generations. Male common voles were housed individually in translucent cages within visual and audible range of each other, with the provision that adjacent cages belonged to different experimental groups. Cages were placed in a sound-attenuated, climate controlled $\left(22 \pm 1^{\circ} \mathrm{C} ; 70 \%\right.$ relative humidity) room, and all voles were initially entrained to $12 \mathrm{~h}$ of dark alternating with $12 \mathrm{~h}$ of light (LD 12:12; $L=250-350$ lux, depending on cage placement, $D=$ dim red light). After the animals were fully entrained, the 12:12 LD cycle was replaced with a single, 30-min light pulse (LP) at the beginning of the subjective night. Entrainment to a single LP was chosen to minimize negative masking of activity (Aschoff, 1960; Mrosovsky, 1999) that could interfere with activity patterns induced by running wheels and temporal food availability (FA). The LP was chosen such that it would lead to stable entrainment of the LEO by phase delays (DeCoursey, 1972; Pittendrigh \& Daan, 1976). Food and light conditions and running-wheel availability varied with the experimental protocols. All experiments were approved by the Animal Experimentation Committee of the University of Groningen (DEC 2809 \& 2809-1) and were performed in agreement with international ethical standards and with the standards of the journal (Portaluppi et al., 2010).

\section{Re-entrainment to a phase advance in light}

Experiment 1 was designed to assess the effect of FA timing on a phase advance of $4 \mathrm{~h}$ in the light entrainment (jet-lag). An additional level of variation was introduced by performing the experiment both on animals housed with and without a running wheel. After initial entrainment in LD 12:12, animals were entrained to a 30-min LP at the beginning of the subjective night for 10 days, after 
which the LP was phase-advanced by $4 \mathrm{~h}$. The effect of FA on entrainment was investigated by removing FA from two groups of animals during the $8 \mathrm{~h}$ following the LP ( $\mathrm{N}=7$ per group). After the phase advance in the LP, the time of restriction either remained at the same clock time (stable FA; $\mathrm{N}=14$ ), or was phase advanced (shifting $\mathrm{FA} ;(\mathrm{N}=14)$ with the LP. Common voles have not been reported to hoard food in nature, or while housed in our laboratory, but they do spill food on the cage bottom while feeding; therefore in the control group $(\mathrm{N}=14)$, where food was available $a d$ libitum, animals did receive a clean cage daily. Because in the absence of a running wheel, the circadian component of activity was anticipated to be weak in some cases, six voles were housed with and eigth voles without a running wheel. From the voles without a running wheel, three voles in each of the groups with FA and two voles in the control group exhibited a circadian activity component that was too weak to be included in a comparative analysis. The eventual group sizes were thus six animals per group, with the exception of $\mathrm{N}=5$ for the two FA groups housed without a running wheel.

Food restriction was limited to $8 \mathrm{hs}$ absence of food. A period of $8 \mathrm{~h}$ would on average comprise three ultradian feeding bouts, and previous work indicated that restricting food for longer than $8 \mathrm{~h}$ leads to weight loss (Van der Veen et al., 2006), which would add an unwanted caloric food restriction to the paradigm. Cage bedding was refreshed every day preceding the food restriction, also for the animals that had ad libitum FA.

\section{Activity components related to light, food, and running-wheel availability}

\section{Behavioral phasing in the presence of timing cues}

Experiment 2 was designed to investigate the contributions of the ultradian activity component and the light and FA-entrained components to overall activity. Animals were again entrained to the 30 min LP every $24 \mathrm{~h}$ for 10 days. After these 10 days, the cycle length $(T)$ of the LP was reduced from $T$ 
$=24$ to $\mathrm{T}=23.5 \mathrm{~h}$. After a period of 10 cycles of the LP with $\mathrm{T}=23.5 \mathrm{~h}$, animals were introduced to a FA-cycle of $8 \mathrm{~h}$ during which FA was restricted and $17 \mathrm{~h}$ of FA $(T=25)$. Two groups of animals were either given the first episode of $8 \mathrm{~h}$ of food restriction starting directly after lights off ( $\mathrm{N}=16)$, i.e., at the beginning of the active phase, or $12 \mathrm{~h}$ later $(\mathrm{N}=16)$. After 16 food restriction cycles, FA was restored to continuous ad libitum availability, while the LP cycle continued. Half of the animals in each group was housed with, and the other half without a running wheel. In this protocol, the phases of the conflicting light and FA cues were subsequently in phase, then moving to anti-phase, and back to being in phase, spanning a whole circadian day. Two animals with and three animals without a wheel did not show sufficient circadian activity to be included in the analysis.

\section{Behavioral phasing in the absence of timing cues}

Besides the acute influence that FA can have on timing of activity, also the sustained (entrained) effects of FA on timing of activity (both phase and period length) were analyzed in the 16 voles which underwent $8 \mathrm{~h}$ of food restriction starting directly after lights off. These voles were again subjected to a $\mathrm{T}=23.5 \mathrm{~h}$ LP cycle, and the same $\mathrm{T}=25 \mathrm{~h}$ food restriction cycle. However, this time, ad libitum FA was restored after 7 FA cycles, and at the same time the cycle in LP was discontinued, allowing animals to free-run from the onset of the $8^{\text {th }}$ cycle FA cycle, when the phase difference between food and light availability would have been maximal.

\section{Behavioral analysis}

Activity was recorded using Passive Infra-Red detectors and stored in 2-min epochs on a PC. Periodicity of activity during entrained and free-running conditions was assessed using periodogram analysis (Sokolove \& Bushell, 1978). For Experiment 1, phases of activity were calculated as a circular Center of Gravity (CG) of activity (Hut et al., 2000; Kenagy, 1980). CGs were calculated according to Mardia (1972) over a 24-h period, starting at each consecutive 2-min epoch in the activity data set 
(running CG). An average CG (and linearized SD) was then calculated for each day, with the criterion that a gap of more than 1000 min between two consecutive CGs indicated a transition between two days. As a correction for baseline inter-individual variation within a group, the CG for each individual was corrected for the difference between the individual average CG during first 10 days and the group CG for that period. Data were then expressed as average daily CG and SEM.

For Experiment 2, the contribution of the ultradian components was estimated by smoothing the data over a 3-h window and subtracting the smoothed data from the raw data. The proportions of the behavior belonging to the two components that appear during the desynchronized T-cycles were assessed using a deconvolution approach according to Hiddinga et al. (1997) on the smoothed data. In short, the data were folded on a 25-h basis, and the circular average profile was subtracted from the raw data. Periodogram analysis of the residual dataset, containing activity that was not accounted for by the 25 -h profile, was used to establish the period of the dominant rhythmic component. The data were then folded on the basis of this second component, and the circular average was subtracted from the raw smoothed data. The residual dataset of this second component was then used to re-assess the period of the first component. This process was iterated three times, at which time the periods of the two rhythmic components were stable. The contribution of the different activity components to the behavior was expressed as the percentage of total variance

All statistical analysis was performed using SAS 9.1, SAS Institute Inc., Cary, NC, USA. 


\section{RESULTS}

\section{Re-entrainment to a phase advance in light}

All voles $(\mathrm{N}=42)$ entrained to the $30-\mathrm{min} \mathrm{LP}$, and periodogram analysis showed entrainment to the light protocol with a mean period of $23.9 \pm 0.04 \mathrm{~h}$ (AVG \pm SEM). Average phase angles of entrainment (time of lights on - time of corrected CG) were calculated over days 6-10 of the prephase advance, and days 6-10 of the post-phase advance period. The phase angles of entrainment are shown in Table 1. Pre-phase shift, phase angles are not different between the treatment groups within the animals housed with or without a running wheel (Proc MIXED, Wheel*Food P $>0.05$ ). The phase angles of entrainment are different between animals housed with and without a running wheel before and after the phase-shift (Proc MIXED, Wheel*Light $P<0.05$ ), and that difference is more than an hour from the pre-phase shift condition (LSM, $P=0.05)$; post light-shift, the differences are also due to the feeding conditions.

Figure 1 shows the behavioral phases as corrected CG for the different conditions before and after the shift in the light pulse. There are differential responses between feeding and housing conditions to the phase shift in the light (Proc MIXED, Wheel*Food*Light $P<0.01$ ). Differences are seen between pre- and post-phase shift in the light, for behavioral phases within a feeding condition, and between feeding conditions during the post-phase shift period. Ad libitum fed voles, both those housed with or without a running wheel, show post-phase shift behavioral phases that are intermediate to those of the voles that receive stable or shifting-food restriction (LSM, Ps $<0.05$ at least). Also similar between voles housed with and without a running wheel, is the enhanced phase advance in behavior when the phase shift in the LP is combined with a similar shift in food restriction (LSM, Ps < 0.001), and the absence of a shift in behavioral phase when their food restriction remains at the same time of day (LSM, Ps > 0.05). 
Voles housed with a running wheel demonstrate a significantly larger behavioral phase advance in response to the phase advance in the light, both in the ad libitum and FA availability conditions (LSM, Ps $<0.05$ at least). In addition to that, in voles that are housed without a running wheel, the behavioral phase is significantly more advanced when the FA advances with the LP (LSM, $\mathrm{P}<0.05$ and $\mathrm{P}<0.0005$ compared to ad libitum and stable FA conditions, respectively). In contrast, voles that are housed with a running wheel, show behavioral phases that are significantly less advanced when the FA remains at the same time of day (LSM, $\mathrm{P}<0.05$ and $\mathrm{P}<0.0005$ to ad libitum and shift in FA conditions, respectively).

\section{Activity components related to light, food, and running wheel availability}

\section{Behavioral phasing in the presence of timing cues}

To further investigate the combined effects of running-wheel availability, LP cycles and temporal food restriction, we applied a protocol of desynchronized T-cycles of LP and FA, and applied it to both animals housed with and without a running wheel.

The ultradian component was assessed using a smoothing paradigm, and the percentage of total variance accounted for by this component (see Table 2) was not different between any of the four groups of voles, housed either with or without a running wheel, receiving the first food restriction following the LP or $12 \mathrm{~h}$ later (Proc MIXED, $\mathrm{P}>0.05$ ). Figure $2 \mathrm{~A}$ shows a representative, double plotted, quantitative actogram of a vole housed with a running wheel receiving the first food restriction directly following the LP. Using a deconvolution method, we assessed the periods of the two major activity components in the circadian range, and calculated the percent of total variance explained by these activity components. The group values for periods and contributions are shown in Table 2, and Figure 2B, 2C, and 2D show the periodograms for the behavioral segments before, during, and after the food restriction cycle for the actogram shown in Figure 2A. The periods of the 
two activity components identified were on average $24.14 \mathrm{~h}(\mathrm{SEM}=0.14 \mathrm{~h})$ for one component, and $25.05 \mathrm{~h}(\mathrm{SEM}=0.01 \mathrm{~h})$ for the other period component. Based on these periods, these components will be referred to below as the shorter- and longer-period components. The periods of these components do not vary within housing condition or within time of first food restriction (Proc MIXED, Ps $>0.05$ for Wheel and Food). The length of the short-period component is more than $0.5 \mathrm{~h}$ longer than that of the LP cycle, while the length of the longer-period activity component is very close to that of the length of the feeding cycle.

The percent of total variance explained by the shorter and longer component (see Figure 2E and $2 \mathrm{~F}$ ) are, in all cases, significantly larger for the longer component (Proc Mixed, Component $\mathrm{P}<$ 0.0001 ), and does not vary with housing or feeding condition (Proc Mixed, Ps $>0.05$ for Wheel and Food). A larger contribution of the longer-period component to the overall activity is also reflected in the higher peak values of the periodogram of that component (Figure $2 \mathrm{C}$ ).

\section{Behavioral phasing in the absence of timing cues}

To assess whether the activity component would continue to be expressed in the absence of the Zeitgebers, we re-ran animals housed with and without a running wheel in the same protocol of the T-cycles of LP and FA, starting the first food restriction immediately after the LP. We stopped both the LP and FA cycles after the $7^{\text {th }}$ FA cycle, so that the last FA and LP timing cues were 180 o out of phase. When released in free-running conditions, the period of the voles was $23.73 \mathrm{~h}$ (SEM $=0.15$ ), which was not significantly different from the shorter-period component in the preceding experiment ( $\mathrm{t}$-test, $\mathrm{P}>0.05$ ). A number of responses were seen, and Figure 3 shows examples of double-plotted actograms of the four types of responses observed. The actogram in Figure 3A shows a bimodal distribution of activity, and both components have a similar periodicity. The unsmoothed activity profile in Figure $3 \mathrm{~B}$ again shows a bimodal distribution, but the smoothed data indicate that the longer-period component is phased similar to the feeding conditions and has a larger contribution at first. However, the activity seems to redistribute to a phase more similar to that of 
the preceding LP cycle. Actograms in Figures $3 \mathrm{C}$ and 3D show only single components in activity. The individual in Figure $3 \mathrm{C}$ shows a single activity component with a period and phase similar to that of the preceding FA cycle, while the individual in Figure 3D shows an activity component with a phase similar to that of the preceding LP cycle. 


\section{DISCUSSION}

\section{Food and running-wheel availability alters the contribution of light to behavioral phasing}

We show that timing of food availably can strongly influence activity patterns in the common vole. When adjusting to a phase advance in light entrainment, FA timing that remains at the same time of day results in a strongly attenuated behavioral re-entrainment response. In contrast, when FA is shifted in accordance with the phase advance in light, behavioral re-entrainment is more pronounced.

Running-wheel availability affects re-entrainment to the new LP in two ways. Firstly, the magnitude of the phase advance in behavior is greater in voles housed with a running wheel in both the ad libitum and temporally restricted FA conditions. Secondly, post-phase shift the effect of FA, as compared to the ad libitum FA conditions, on behavioral phasing is opposite between voles housed with and without a running wheel. While in voles housed without a running wheel the behavioral phase advance is significantly increased by shifting FA together with the LP, in voles housed with a running wheel a significantly decreased behavioral response is seen when FA remains at the same time of day. These effects may be opposite sides of the same coin, and indicate that the impact of light as a timing cue is greater when behavioral organization is more circadian, and that impact of food as a timing cue is more emphasized in ultradian individuals.

This work adds a behavioral equivalent to our previously published work, showing the molecular circadian clock in the liver (which is sensitive to food availability) is inactive in voles with a predominant ultradian behavioral pattern, but is active in voles with circadian organization of behavior (Van der Veen et al., 2006). The result on the relative contribution of the LP and FA in the timing of behavior was most likely also dependent on the protocol. A brief light pulse per cycle may be sufficient for circadian entrainment, but is a weak Zeitgeber compared to a daily 8-h food- 
deprivation episode. A single light pulse has been shown to be sufficient to entrain circadian rhythms (DeCoursey, 1972; Pittendrigh \& Daan, 1976), in our case the pulse was directed to fall at the end of the inactive phase. It has, however, been shown that multiple light pulses in the dark period can lead to stress in the social vole, Microtus socialis (Zubidat et al., 2007). Apart from the single light pulse each cycle, animals were kept in dim red light. Dim light during the dark phase, instead of darkness, has been shown to increase the speed of re-entrainment in hamsters (Evans et al., 2009), and while spectral sensitivity to red light is low, a single red LED in a movement detector has been shown to lengthen the free-running period of mice (Hofstetter et al., 2005).

The arousing effects of cage cleaning may have had an entraining property in our experiment, and may have altered phasing of activity. Phasing of activity in the control group is, however, intermediate to that of either FA groups, indicating that phasing may have been different from non-cage cleaned conditions, but the effects of FA remain. The phasing of the FA cycle may also have had an effect on its relative strength. Kalsbeek et al. (2000) have shown that a 2-h FA during the light phase attenuated re-entrainment of pineal melatonin release to an $8 \mathrm{~h}$ phase advance in light in rats. The FA in Kalsbeek's study, however, was given during the light phase and induced activity at a circadian rest phase, while we restricted FA to the dark phase.

How the presence of a running wheel enhances the circadian component in activity is unclear, but is possible it does so without altering the LEO. Running-wheel availability increases the diminished circadian structuring of activity in mice with diminished SCN cellular synchronization without altering transcriptional activity in the SCN (Power et al., 2010). Indeed, in voles, the amplitude of transcriptional activity is decreased under constant dim light, when activity patterns are diminished (Van der Veen et al., 2008). Both seem to indicate that the SCN output is weak in cases where running-wheel availability can alter circadian amplitude. Indeed, we have shown previously that SCN output (vasopressin), but not core output, (measured at the level of VIP) seems 
altered in voles with weak rhythms (Jansen et al., 2007) and that the cause of these differences may even lie outside the SCN (Van der Veen et al., 2005).

\section{Light and food can drive separate components of behavior}

We observed three components in activity during the combined light and food entrainment paradigm, an ultradian component and two circadian components. Of the circadian components, the component with a period and phasing similar to that of the food cycle was the most dominant. A less dominant component had a period close to $24 \mathrm{~h}$ and did not seem to be entrained by the LP. Thus, while the restricted FA seems to act as an entraining signal, light could no longer entrain under these conditions. It has been shown that rats can entrain to multiple Zeitgebers (for reviews see (Mistlberger, 2009; Stephan, 2002)). Similar to our results in the vole, in rats a free-running component in activity, besides a food entrained component, has also been reported in experiments where no light cue was available (Edmunds \& Adler, 1977). While the LP was strong enough to entrain activity alone, perhaps when combined with restricted FA the signal is no longer strong enough to entrain. Although the short-period component was not driven by light, its period, initial phase, and co-expression next to the food-related component seem to indicate that it was driven by the LEO.

\section{Light and food entrainable oscillators}

The design of these experiments primarily addressed the timing of activity to light and food availability and the role of activity in this process. In the last experiment, we examined whether the activity components could be driven by separate oscillators. When released from their entraining conditions, with food and light in anti-phase, voles showed different behavioral responses. Animals either displayed a bimodal circadian activity distribution, a continuation of the distribution seen 
during the entrainment by two timing systems, or only a single component, either originating from the light or from food entrained activity. Some voles in free-running conditions initially showed a dominant, food-related component, and then shifted to a phase similar to the prior shorter activity component.

A food-entrained component in behavior is known to disappear rather quickly in ad libitum conditions (Stephan et al., 1979), and Coleman et al. (1982) reported that in rats, several weeks after a FA protocol an activity bout was still discerned at the phase of the former feeding time. When those same rats were given episodes of ad libitum food availability this component in behavior disappeared, only to re-appear in a subsequent episodes of food deprivation, although shifting towards and eventually merging with the activity component belonging to the former LP. While rhythms in liver clock genes are normally expressed in mice and rats (Damiola et al., 2000; Stokkan et al., 2001), in voles they are only present under food-restriction cycles or when provided with a running wheel (Van der Veen et al., 2008). The circadian (as opposed to ultradian) feeding pattern induced by the availability of a running wheel and/or restricted food availability likely alters the entrainment of the FEO. The state of this oscillator may well be related to oscillations of the clock in the liver, and also contribute to the effect that food restriction has on phasing of activity in these experiments. In all cases, the SCN is unaffected by restricted FA, unless at the same time this restricts the amount of food consumption (Mendoza et al., 2005). Thus, while temporal food availability is a potent timing cue, it does not normally act at the level of the central pacemaker, but at the level of behavior and peripheral clocks. Although it is uncertain to what degree the foodassociated component was driven by an oscillator structure in our experiments, it was able to alter the light-entrained, SCN-driven activity component, both at the levels of phase and period.

In nature, the herbivorous vole is unlikely to be faced with diurnal rhythms in FA, but rather will be exposed to varying predation risks over the day when venturing out to forage (Gerkema, 2002). Both predator activity and the vole response to this predation risk (e.g. social synchronization 
of feeding) are dependent on entrained circadian rhythms (Gerkema \& Verhulst), and vole ultradian activity patterns show circadian modulation (Gerkema et al., 1993). The common vole is the most widespread Eurasian species of open grasslands (Niethammer \& Knapp 1982). In nature the importance of alignment of light-entrained behavior with other external temporal cues will prevail, tuning the ultradian activity patterns to the optimal circadian phase.

Light-entrained activity can be effectively challenged by FA and behavioral modulation. Firstly, we show that running-wheel availability enhances the entraining potential of light and of FA. Secondly, we show that challenging behavior that is driven by a light cycle with a second cycle in FA alters the entrainment to the light cycle. Moreover, our data indicate that the activity component previously entrained to the light, likely is no longer entrained and possibly driven by a free-running light-entrainable clock. In our data, activity has been shown to be a complex of individually driven components, and this activity is, itself, an important modulator of Zeitgeber effectiveness.

\section{Declaration of interest}

The authors report no conflicts of interest 


\section{REFERENCES}

Aschoff J. (1960). Exogenous and endogenous components in circadian rhythms. Cold Spring Harb. Symp. Quant. Biol. 25:11-28.

Castillo MR, Hochstetler KJ, Tavernier RJ Jr., Greene DM, Bult-Ito A. (2004). Entrainment of the master circadian clock by scheduled feeding. Am. J. Physiol. Regul. Integr. Comp. Physiol. 287:R551-R555.

Coleman GJ, Harper S, Clarke JD, Armstrong S. (1982). Evidence for a separate meal-associated oscillator in the rat. Physiol. Behav. 29:107-115.

Daan S, Slopsema S. (1978). Short-term rhythms in foraging behavior in the common vole, Microtus arvalis. J. Comp. Physiol .[A] 127:215-227.

Damiola F, Le MN, Preitner N, Kornmann B, Fleury-Olela F, Schibler U. (2000). Restricted feeding uncouples circadian oscillators in peripheral tissues from the central pacemaker in the suprachiasmatic nucleus. Genes Dev. 14:2950-2961.

DeCoursey PJ. (1972). LD ratios and the entrainment of circadian activity in a nocturnal and a diurnal rodent. .J Comp. Physiol. [A] 78:221-235.

Edgar DM, Dement WC. (1991). Regularly scheduled voluntary exercise synchronizes the mouse circadian clock. Am. J. Physiol .261:R928-R933.

Edmunds SC, Adler NT. (1977). The multilicity of biological oscillators in the control of circadian running activity in the rat. Physiol. Behav. 18:921-930.

Evans JA, Elliott, JA, Gorman, MR. (2009). Dim nighttime illumination accelerates adjustment to timezone travel in an animal model. Curr. Biol. 19:R156-R157.

Gerkema MP. (2002). Ultradian rhythms. In Kumar V (ed). Biological Rhythms. New Delhi: Narosa Publ, pp. 207-215.

Gerkema MP, Van der Leest LF. (1991). Ongoing ultradian activity rhythms in the common vole, Microtus arvalis, during deprivations of food, water and rest. J. Comp. Physiol. A 168:591597.

Gerkema MP, Verhulst S. (1990). Warning against an unseen predator: an experimental study in the common vole, Microtus arvalis. J. Anim. Behav. 10:1169-1178.

Gerkema MP, Groos GA, Daan S. (1990). Differential elimination of circadian and ultradian rhythmicity by hypothalamic lesions in the common vole, Microtus arvalis. J. Biol. Rhythms 5:81-95.

Gerkema MP, Daan S, Wilbrink M, Hop MW, Van der Leest F. (1993). Phase control of ultradian feeding rhythms in the common vole (Microtus arvalis): the roles of light and the circadian system. J. Biol. Rhythms 8:151-171.

Hiddinga AE, Beersma DG, Van den Hoofdakker RH. (1997). Endogenous and exogenous components in the circadian variation of core body temperature in humans. J. Sleep Res. 6:156-163. 
Hofstetter JR, Hofstetter AR, Hughes AM, Mayeda AR. (2005). Intermittent long-wavelength red light increases the period of daily locomotor activity in mice. . Circadian Rhythms 3:8.

Hut RA, Scheper A, Daan S. (2000). Can the circadian system of a diurnal and a nocturnal rodent entrain to ultraviolet light? J. Comp. Physiol. A 186:707-715.

Jansen K, Van der Zee EA, Gerkema MP. (2007). Vasopressin immunoreactivity, but not vasoactive intestinal polypeptide, correlates with expression of circadian rhythmicity in the suprachiasmatic nucleus of voles. Neuropeptides 41:207-216.

Kalsbeek A, Barassin S, Van Heerikhuize JJ, Van der Vliet J, Buijs RM. (2000). Restricted daytime feeding attenuates reentrainment of the circadian melatonin rhythm after an 8-h phase advance of the light-dark cycle. J. Biol. Rhythms 15:57-66.

Kenagy GJ. (1980). Center-of-gravity of circadian activity and its relation to free-running periods in two rodent species. J. interdiscipl Res. 11:1-8.

Krieger DT, Hauser H, Krey LC. (1977). Suprachiasmatic nuclear lesions do not abolish food-shifted circadian adrenal and temperature rhythmicity. Science 197:398-399.

Marchant EG, Mistlberger RE. (1996). Entrainment and phase shifting of circadian rhythms in mice by forced treadmill running. Physiol. Behav. 60:657-663.

Mardia, K. V. (1972) Statistics of directional data. In Academic press, inc., London.

Mendoza J, Graff C, Dardente H, Pevet P, Challet E. (2005). Feeding cues alter clock gene oscillations and photic responses in the suprachiasmatic nuclei of mice exposed to a light/dark cycle. J. Neurosci. 25:1514-1522.

Mistlberger RE. (1991). Effects of daily schedules of forced activity on free-running rhythms in the rat. J. Biol. Rhythms 6:71-80.

Mistlberger RE. (1993). Effects of scheduled food and water access on circadian rhythms of hamsters in constant light, dark, and light:dark. Physiol . Behav. 53:509-516.

Mistlberger RE. (2009). Food-anticipatory circadian rhythms: concepts and methods. Eur. J. Neurosci. 30:1718-1729.

Mistlberger RE, Holmes MM. (2000). Behavioral feedback regulation of circadian rhythm phase angle in light-dark entrained mice. Am. J. Physiol. Regul. Integr. Comp. Physiol. 279:R813-R821.

Moore RY, Eichler VB. (1972). Loss of a circadian adrenal corticosterone rhythm following suprachiasmatic lesions in the rat. Brain Res. 42:201-206.

Mrosovsky N. (1988). Phase response curves for social entrainment. J. Comp. Physiol. A 162:35-46.

Mrosovsky N. (1999). Masking: history, definitions, and measurement. Chronobiol. Int. 16:415-429.

Mrosovsky N, Salmon PA. (1987). A behavioral method for accelerating re-entrainment of rhythms to new light-dark cycles. Nature 330:372-373.

Niethammer J, Knapp F. (1982). Handbuch der Saeugetiere Europas Band 2-1, Akademische Verlaggesellschaft, Wiesbaden. 
Pittendrigh CS, Daan S. (1976). A functional analysis of circadian pacemakers in nocturnal rodents V. Pacemakers structure: a clock for all seasons. J. Comp. Physiol. [A] 106:333-355.

Portaluppi F, Smolensky MH, Touitou Y. (2010). Ethics and methods for biological rhythm research on animals and human beings. Chronobiol . Int. 27:1911-1929.

Power A, Hughes ATL, Samuels RE, Piggins HD. (2010). Rhythm-promoting actions of exercise in mice with deficient neuropeptide signalling. J. Biol. Rhythms 25:235-246.

Ralph MR, Foster RG, Davis FC, Menaker M. (1990). Transplanted suprachiasmatic nucleus determines circadian period. Science 247:975-978.

Reebs SG, Lavery RJ, Mrosovsky N. (1989). Running activity mediates the phase-advancing effects of dark pulses on hamster circadian rhythms. J. Comp. Physiol. A 165:811-818.

Sokolove PG, Bushell WN. (1978). The chi square periodogram: its utility for analysis of circadian rhythms. J. Theor. Biol. 72:131-160.

Stephan FK. (1986). Interaction between light- and feeding-entrainable circadian rhythms in the rat. Physiol. Behav. 38:127-133.

Stephan FK. (2002). The "other" circadian system: food as a Zeitgeber. J.Biol.Rhythms 17:284-292.

Stephan FK, Zucker I. (1972). Circadian rhythms in drinking behavior and locomotor activity of rats are eliminated by hypothalamic lesions. Proc. Natl. Acad. Sci. U.S.A 69:1583-1586.

Stephan FK, Swann JM, Sisk CL. (1979). Entrainment of circadian rhythms by feeding schedules in rats with suprachiasmatic lesions. Behav. Neural. Biol. 25:545-554.

Stokkan KA, Yamazaki S, Tei H, Sakaki Y, Menaker M. (2001). Entrainment of the circadian clock in the liver by feeding. Science 291:490-493.

Van der Veen DR, Castillo MR, van der Zee EA, Jansen K, Gerkema MP, Bult-Ito A. (2005). Circadian dynamics of vasopressin in mouse selection lines: translation and release in the SCN. Brain Res 1060:16-25.

Van der Veen DR, Minh NL, Gos P, Arneric M, Gerkema MP, Schibler U. (2006). Impact of behavior on central and peripheral circadian clocks in the common vole Microtus arvalis, a mammal with ultradian rhythms. Proc.Natl.Acad.Sci.U.S.A 103:3393-3398.

Van der Veen DR, van der Pol-Meijer MM, Jansen K, Smeets M, van der Zee EA, Gerkema MP. (2008). Circadian rhythms of C-FOS expression in the suprachiasmatic nuclei of the common vole (Microtus arvalis). Chronobiol.Int. 25:481-499.

Zubidat AE, Ben-Shlomo R, Haim A. (2007). Thermoregulation and endocrine repsonses to light pulses in short-day acclimated social voles (Microtus socialis). Chronobiol. Int. 24: 269-288. 


\section{TABLES}

Table 1: Phase angles of entrainment pre- and post-phase shift in the light

Phase angle between light and behavioral phase

\begin{tabular}{llcc} 
& Food availability & $\begin{array}{c}\text { Pre-phase advance } \\
\text { Mean } \pm \text { SEM }\end{array}$ & $\begin{array}{c}\text { Post-phase advance } \\
\text { Mean } \pm \text { SEM }\end{array}$ \\
\hline \multirow{2}{*}{ No wheel } & Ad libitum & $-2.40 \pm 0.36 \mathrm{~h}$ & $-5.33 \pm 0.51 \mathrm{~h}$ \\
& Shifting with light & $-2.75 \pm 0.21 \mathrm{~h}$ & $-7.23 \pm 0.16 \mathrm{~h}$ \\
& Remaining at same time & $-2.92 \pm 0.19 \mathrm{~h}$ & $-2.75 \pm 0.42 \mathrm{~h}$ \\
\multirow{2}{*}{ Wheel } & Ad libitum & $-1.50 \pm 0.25 \mathrm{~h}$ & $-1.92 \pm 0.58 \mathrm{~h}$ \\
& Shifting with light & $-1.40 \pm 0.17 \mathrm{~h}$ & $-4.24 \pm 0.42 \mathrm{~h}$ \\
& Remaining at same time & $-1.55 \pm 0.22 \mathrm{~h}$ & $-0.53 \pm 0.45 \mathrm{~h}$
\end{tabular}


Table 2: Activity components identified under desynchronized T-cycles

\begin{tabular}{|c|c|c|c|c|c|c|}
\hline & \multirow[b]{2}{*}{$\begin{array}{l}\text { Start of food } \\
\text { restriction }\end{array}$} & \multirow{2}{*}{$\begin{array}{l}\text { Ultradian component } \\
\% \text { of total variance } \\
\text { Mean } \pm \text { SEM }\end{array}$} & \multicolumn{2}{|c|}{ First circadian component } & \multicolumn{2}{|c|}{ Second circadian component } \\
\hline & & & $\begin{array}{c}\text { Period } \\
\text { Mean } \pm \text { SEM }\end{array}$ & $\%$ of circadian variance & $\begin{array}{c}\text { Period } \\
\text { Mean + SEM }\end{array}$ & $\%$ of circadian variance \\
\hline \multirow[t]{2}{*}{ No wheel } & After LP & $26.9 \pm 4.6 \%$ & $25.07 \pm 0.02 \mathrm{~h}$ & $35.0 \pm 4.8 \%$ & $24.30 \pm 0.32 \mathrm{~h}$ & $12.2 \pm 1.8 \%$ \\
\hline & $12 \mathrm{~h}$ after LP & $21.3 \pm 2.6 \%$ & $25.01 \pm 0.01 \mathrm{~h}$ & $32.9 \pm 9.5 \%$ & $24.40 \pm 0.44 h$ & $15.3 \pm 4.2 \%$ \\
\hline \multirow[t]{2}{*}{ Wheel } & After LP & $30.8 \pm 3.8 \%$ & $25.03 \pm 0.02 h$ & $31.2 \pm 6.5 \%$ & $24.00 \pm 0.24 h$ & $15.2 \pm 1.2 \%$ \\
\hline & $12 \mathrm{~h}$ after LP & $30.1 \pm 2.6 \%$ & $25.06 \pm 0.02 \mathrm{~h}$ & $23.8 \pm 4.5 \%$ & $23.91 \pm 0.20 h$ & $12.5 \pm 2.2 \%$ \\
\hline
\end{tabular}




\section{FIGURE LEGENDS}

Figure 1

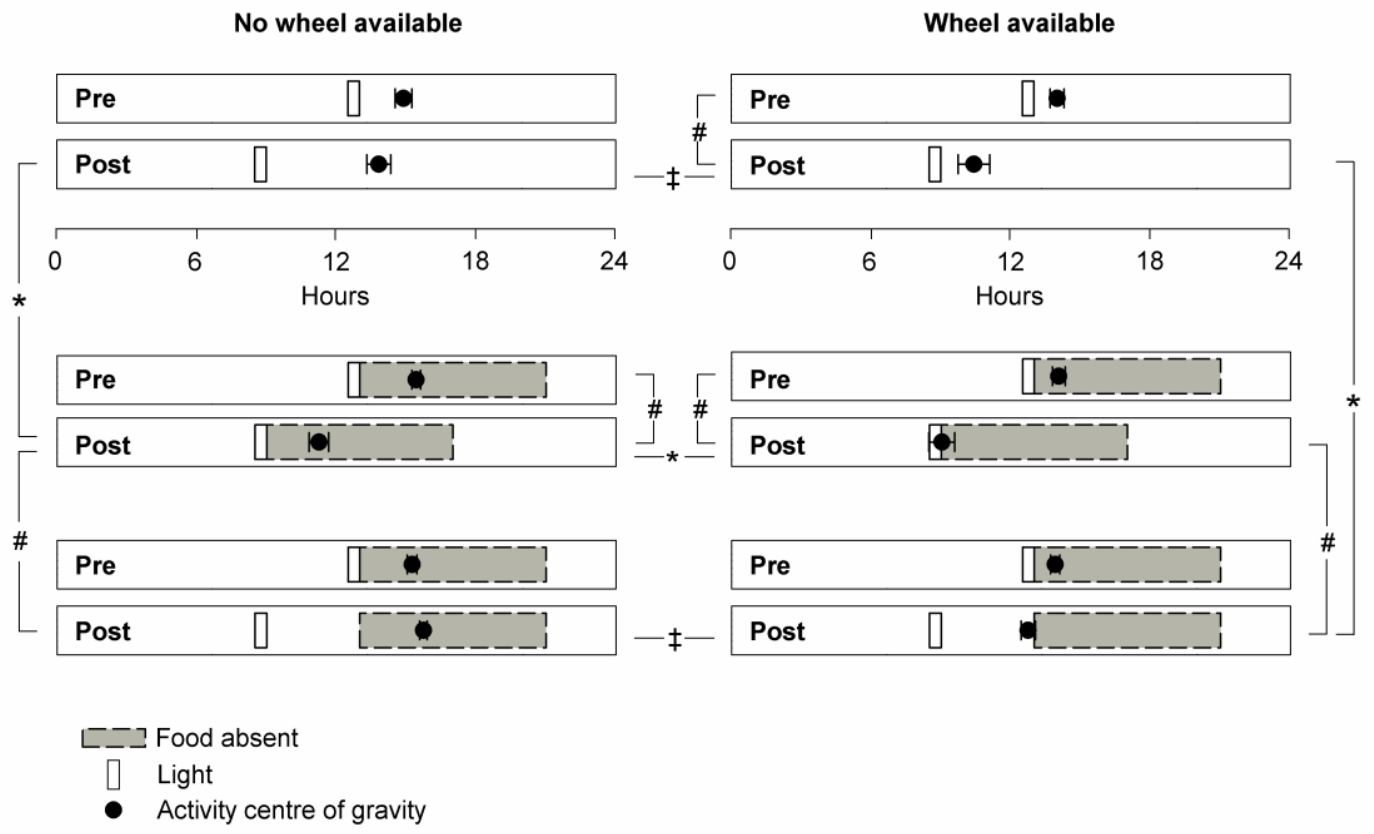

Relative centers of gravity of activity are shown for the last 5 days before a 4-h phase advance in light (Pre), and days 6-10 after the phase advance in the light (Post). Behavioral phases are shown for animals housed without (right panels), and with (left panels) a running wheel. When an 8-h foodrestriction phase advances together with the light, the behavioral phase advance is enhanced in animals housed without a running wheel. The behavioral phase shift to the same phase advance in light is attenuated in voles housed with a running wheel, when food restriction remains at the same time of day. For all three food availability conditions, animals housed with a running wheel show a greater behavioral response to the phase advance in the light than animals housed without a running wheel do. Error bars are SEM, white boxes indicate the 30-min light pulse, grey bars depict the 8-h period when food was absent in the groups that received food restriction. ${ }^{*} P<0.05, \ddagger P<$ $0.005, \# \mathrm{P}<0.0005$ 

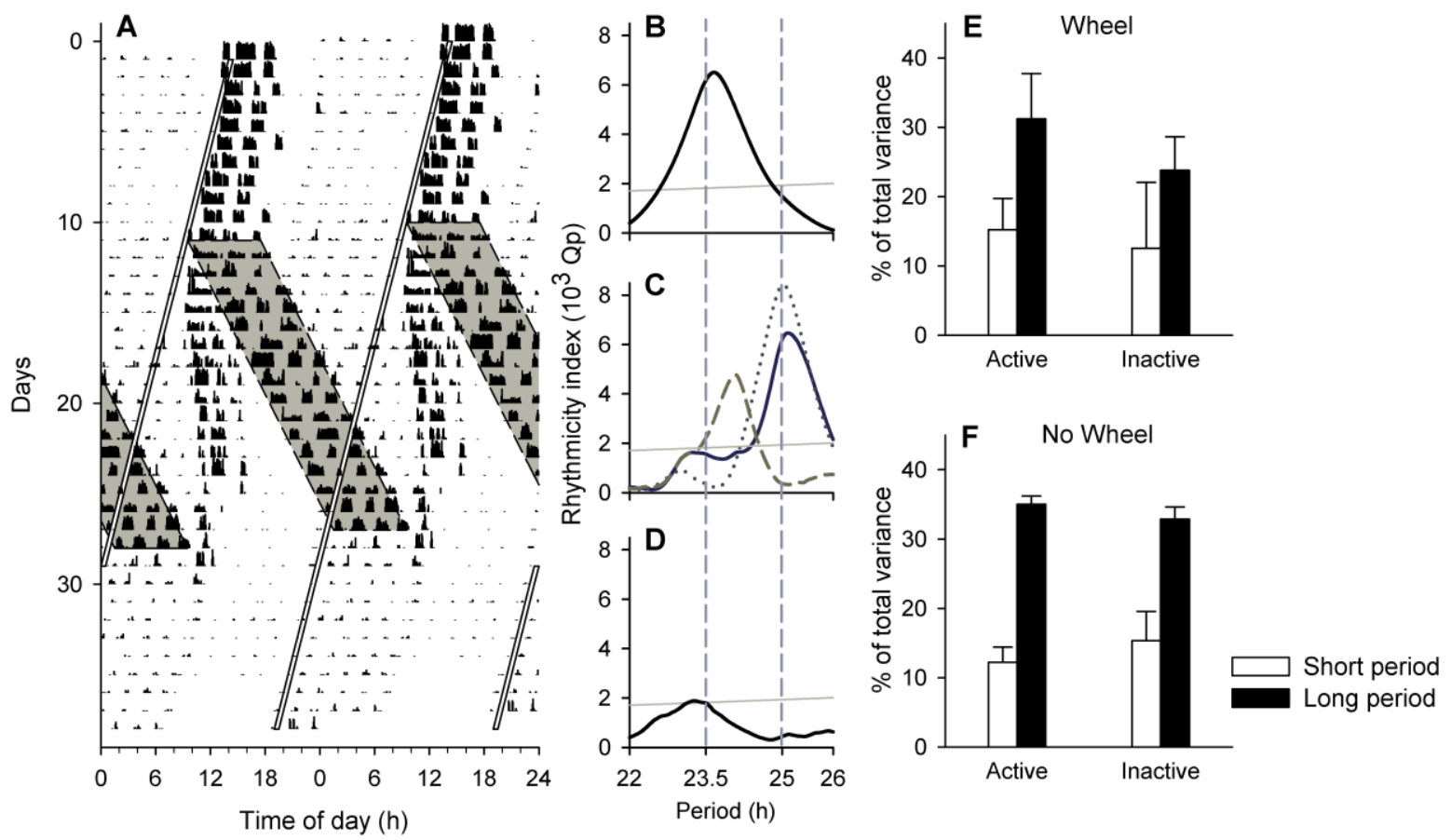

Double-plotted, quantitative actogram of an individual vole housed with a running wheel during entrainment to a single, 30-min light pulse every $23.5 \mathrm{~h}(\mathrm{~A})$. Starting on day 11 , the vole is subjected to 16 episodes of $8 \mathrm{~h}$ food restriction and $17 \mathrm{~h}$ food availability ( $\mathrm{T}=25 \mathrm{~h}$ ); thereafter, food is restored to ad libitum availability. Panels B, C, and D show periodograms of behavioral data before, during, and after the food restriction cycles. When both cycles of light and food availability are presented, the total amount of variance explained by the two major circadian activity components is shown for voles housed with (E) and without (F) a running wheel. In panel A, white line indicates the 30-min light pulse, grey bars depict the 8-h period when food was absent. 
Figure 3

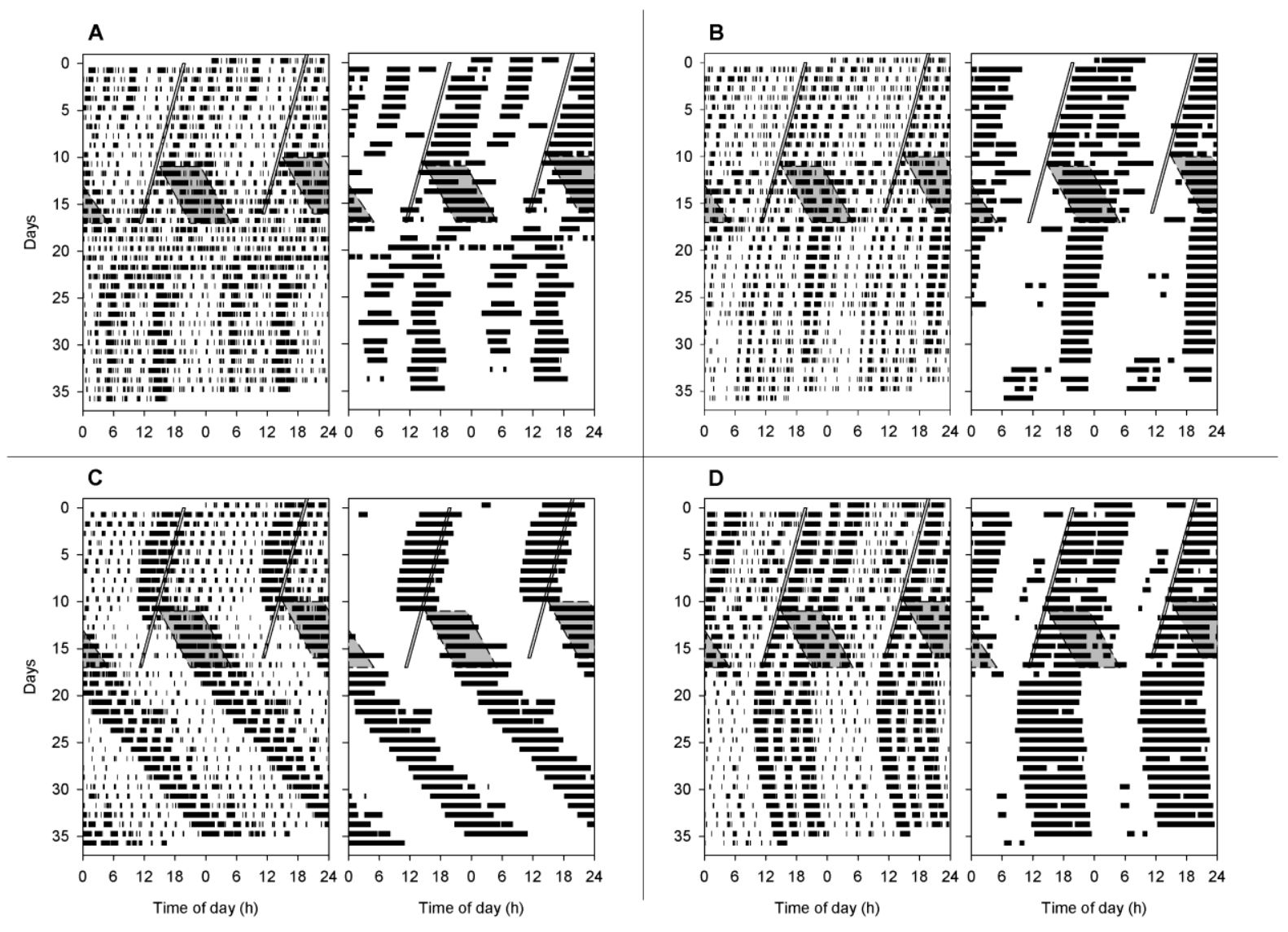

Example of double-plotted actograms of voles subjected to a single, 30-min light pulse every $23.5 \mathrm{~h}$. Starting on day 12 , voles are subjected to 7 episodes of $8 \mathrm{~h}$ food restriction and $17 \mathrm{~h}$ food availability ( $\mathrm{T}=25 \mathrm{~h}$ ); thereafter, food is restored to ad libitum availability, and the cycle in the light pulses is discontinued. For each of the individuals, the total (left) and above average (right) activity is shown. Actograms are representative for the four different responses observed: A bimodal activity distribution (A), or single activity components (B-D). For the individuals showing a single activity component, phasing may shift (B), or remain at the phase of previous food availability (C) or the light pulse (D) cues. White line indicates the 30-min light pulse, grey bars depict the 8-h period when food was absent. 